\title{
Hyperpigmented LipCorner; An Unusual presentation of Acanthosis Nigricans: A Case Report
}

\author{
Filiz Cebeci ${ }^{1}$, Ekin Ozge Ozdemir ${ }^{2}$, Sirin Yasar ${ }^{3}$, Sema Aytekin ${ }^{3}$ and Havva Keskin ${ }^{4 *}$ \\ ${ }^{1}$ Department of Dermatology, Istanbul Medeniyet University Goztepe Training and Research Hospital, Turkey \\ ${ }^{2}$ Department of Dermatology, Ministry of Health University Istanbul Sisli Hamidiye Etfal Education and Research Hospital, Turkey \\ ${ }^{3}$ Department of Dermatology, Ministry of Health University Istanbul Haydarpasa Education and Research Hospital, Turkey \\ ${ }^{4}$ Department of Internal Medicine, Istanbul Medeniyet University Goztepe Training and Research Hospital, Turkey
}

Submission: February 06, 2018; Published: February 23, 2018

*Corresponding author: Havva Keskin, Department of Dermatology, Istanbul Medeniyet University Goztepe Training and Research Hospital, Specialist of Dermatology, Goztepe Mahallesi, Fahrettin Kerim Gokay Cd., 34730, Kadikoy/Istanbul, Turkey, Tel: +90 (505) 7313046; Fax: +90 (216) 5664000; Email: havva.drkeskin@mail.com

\begin{abstract}
Acanthosisnigricans is a fairlycommon skin pigmentationdisorder in obesepeople. This lesi on may be a sign of a moreserious health problem such as metabolic syndrome and some of themalignancy. Over the past several decades, while obesity and metabolic syndrome were a growing health problem across the developed countries, we need to be more cautious and alert about this lesions in routine clinical practice at internal medicine clinics. This case highlights the importance of AN on thelipcorners where AN is rarelylocated.
\end{abstract}

Keywords: Unusual presentation; Acanthosis Nigricans; Obesity

\section{Introduction}

Acanthosisnigricans (AN) is one of the common dermatologic manifestations of obesity and insulinresistance. These lesions are characterized thickenin gand the hyperpigmented darkened lesion and they are located in the flexuralare as of the skin or on themucosal surfaces. These darkened lesions are the typical settlement symmetric in axilla, neck, andgroin. Besides obesity

\section{Case Presentation}

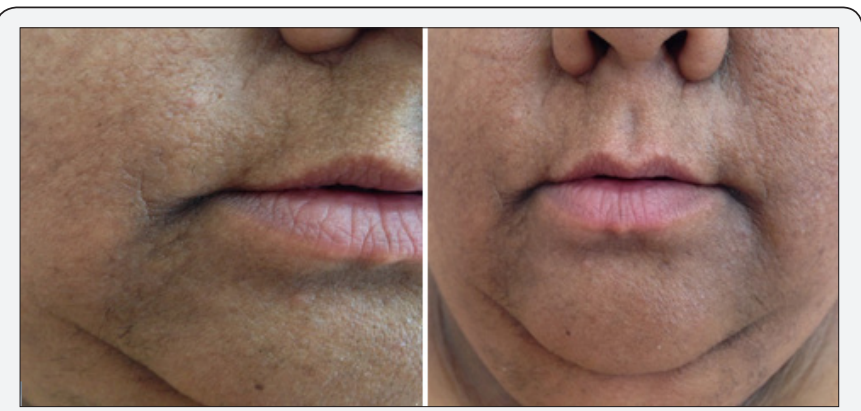

Figure 1: Anterior view of the lip corners. and endocrine syndromes, the other common reasons for AN are genetic, auto immune, paraneo plastic conditions, some medical treatment, andi diopathic [1,2]. In this report, we present a patient with AN who has type 2 diabetes, hypertension, hyperlipidemia, andobesity. Her lesions were described on the neckand on the bilateral lip corners where AN is not usually located.

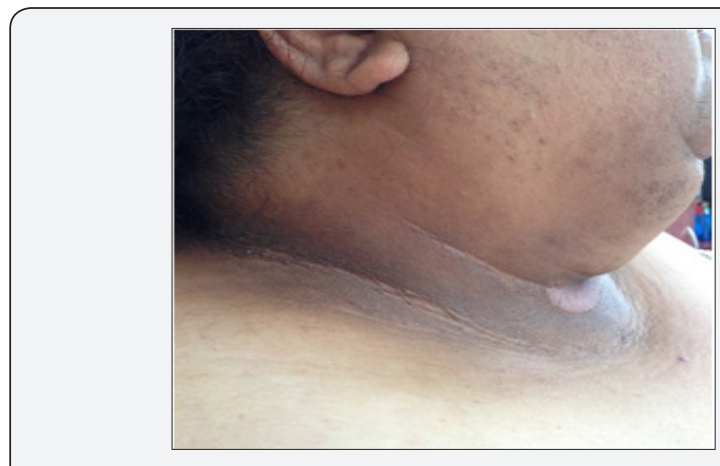

Figure 2 : Lateral view of the neck. 
In this case report, a 56-year-old woman with the hyper pigmented, thicken in glesionson theneck and on the bilateral lip corners was presented for an evaluation. Her lesions were first recognized almost one-year ago, and various types of topical creams were used for treatment. Her examination report revealed that she is obese (body mass index: $32 \mathrm{~kg} / \mathrm{m}^{2}$ ) and there were hirsutism, a general increase in pigmentation throughout the body, thickening skin by the brown-velvety appearance of the lip corners and the neck (Figure $1 \& 2$ ). The similar skin lesions were present in her inter triginous regions. A can thos is and marked papillomatos is were detected by biopsy.

She didn't have the history of type 2 diabetes, hypertension, hyperlipidemia, and malignan this tory before. Her an thropometric measures and metabolic evaluation were compatible with the metabolic syndrome, type 2 diabetes, hypertension, and hyper lipidemia. A pathology that would suggest malignancy was not detected. She was referred to internal medicine department to evaluate her treatment and modify as needed. Her treatment plan was included treatment of type 2 diabetes, hypertension, hyperlipidemia, and the institution of weight loss measures.

\section{Discussion}

Over the past several decades, while obesity and metabolic syndrome were a growing health problem across the developed countries, obesity-related co-morbidities such as metabolic, cardiovascular, carcinogenic, musculo skeletal and cutaneous disorders have been increased. AN is related to cancer as well as obesity, and endocrined is orders including diabetes mellitus, hyper insulinemia, insulin resistance, and metabolic syndrome $[3,4]$. AN may be a sign of some type of cancer when it is evident in an obese person. Therefore, when AN is diagnosed, along with metabolic disorders, malignancy should be eliminated too. This case didn't have any malignant lesion, but she had metabolic syndrome and insulin resistance (type 2 diabetes).

AN can be observed in the non classic locations such as the face, eyelid, circumference of the umbilicus, palmo plantar region, external genital area, breast are ola and mucosal involvement as we observed in our case [5]. This case highlights the importance of AN on the lip corners where AN is rarely located. This case shows that AN can be located in non classical regions on the skin or mucosal surface and we need to be more cautious and alert in routine clinical practice at internal medicine clinics.

\section{References}

1. Li J, Yazdabadi A, Sinclair R (2012) Acanthosis nigricans associated with morbid obesity and colorectal adenocarcinoma. Case Rep Dermatol Med 2012: 545247.

2. Yosipovitch G, DeVore A, Dawn A (2007) Obesity and the skin: skin physiology and skin manifestations of obesity. J Am Acad Dermatol 56(6): 917-920.

3. Niaz F, Shams N, Qureshi S, Bashir F, Shaikh Z, et al. (2015) Dermatological manifestations of obesity. Journal of Pakistan Association of Dermatologists 25(2): 90-95.

4. Hermanns Le T, Scheen A, Pierard GE (2004) A canthos is nigricans associated with insulin resistance: patho physiology and management. Am J Clin Dermatol 5(3): 199-203.

5. Shipman AR, Millington GW (2011) Obesity and the skin. Br J Dermatol 165(4): 743-750.

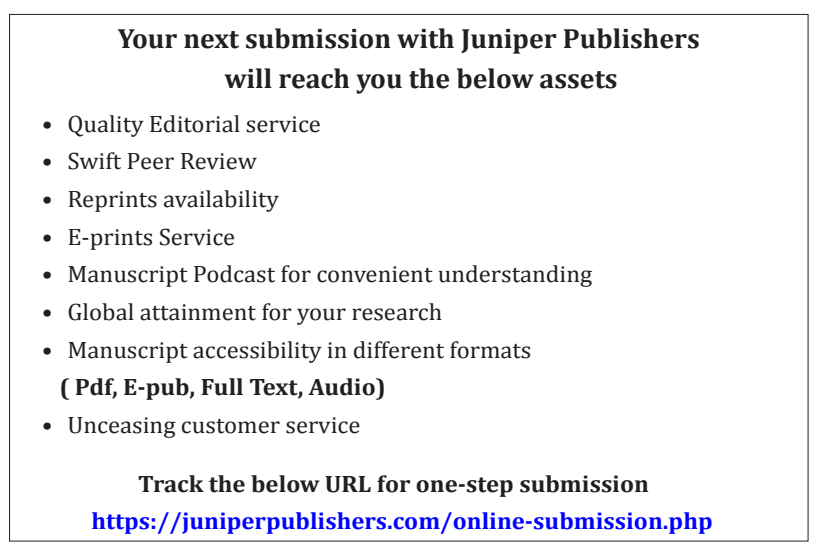

